Journal of Experimental and Clinical Medicine https://dergipark.org.tr/omujecm

Case Report

J Exp Clin Med

2021; 38(3): 379-380

doi: $10.52142 /$ omujecm.38.3.32

\title{
Lance Adams syndrome following post-hypoxic myoclonic status epilepticus in an adolescent patient with chronic renal failure: A case report
}

\author{
Muhammed ÜDÜRGÜCÜ ${ }^{1, * i[}$, Nazik AŞILIOĞLU YENER ${ }^{1}$,
}

${ }^{1}$ Department of Pediatric Critical Care, Faculty of Medicine, Ondokuz Mayıs University, Samsun, Turkey

${ }^{2}$ Department of Pediatry, Faculty of Medicine, Ondokuz Mayıs University, Samsun, Turkey

\begin{abstract}
\begin{tabular}{ccccc}
\hline Received: 27.03 .2020 & $\bullet$ & Accepted/Published Online: 01.02.2021 & $\bullet$ & Final Version: 23.04.2021 \\
\hline
\end{tabular}
\section{Abstract}

Myoclonic contractions that may occur after hypoxia might be related to post-hypoxic status epilepticus (PHSE) or Lance Adams syndrome (LAS). It is important to distinguish these two myoclonic conditions in terms of treatment and prognosis. In the literature, both PHSE and LAS have not been reported in pediatric age group including adolescents. Here we report an adolescent case with chronic renal failure who developed PHSE and then LAS after a long-term cardiopulmonary resuscitation.
\end{abstract}

Keywords: Lance Adams syndrome, posthypoxic status epilepticus, myoclonus, movement disorders, cardiopulmonary arrest

\section{Introduction}

Post-hypoxic myoclonia (PHM) following a cardiac arrest and hypoxia can occur in two different ways, acute and chronic. Acute PHM, called post-hypoxic myoclonic status epilepticus (PHSE), occurs early in patients with severe coma after a cardiac arrest. Chronic PHM, called Lance Adams syndrome (LAS), is characterized by increased myoclonies with stimulations and may occur in patients with significant improvement in consciousness after cardiac arrest. It is important to distinguish LAS from acute PHM. Although it is rarely reported in the literature as case reports in adults, not been reported in childhood. We presented an adolescent case that developed PHSE and then LAS.

\section{Case report}

A 16-year-old male patient who was with the diagnosis of rapidly progressive glomerulonephritis was brought after a cardiopulmonary arrest. The first treatment was given because of the blood potassium level reached $9 \mathrm{mEq} / \mathrm{L}$, then sent to our hospital for hemodialysis. During the transport, he suffered a cardiac arrest and was brought to our hospital after approximately 40 minutes of resuscitation. He was intubated and unconscious and Glasgow Coma Scale (GCS) was 4. arterial blood pressure was $130 / 65 \mathrm{mmHg}$, heart rate was $137 /$ minute, fever was $36.8^{\circ} \mathrm{C}$ degrees.

In laboratory values, $\mathrm{pH}: 6.98, \mathrm{HCO}: 10.6 \mathrm{mmol} / \mathrm{L}$, $\mathrm{pCO}_{2}: 57.2 \mathrm{mmHg}$, lactate:7.2 mmol/L, blood urea nitrogen: 99 $\mathrm{mg} / \mathrm{dL}$, creatine: $12 \mathrm{mg} / \mathrm{dL}$, potassium:9.4 mEq/L, uric acid:9.9 $\mathrm{mg} / \mathrm{dL}$. The patient underwent hemodialysis in the pediatric intensive care unit. At the $18^{\text {th }}$ hour after resuscitation, generalized multifocal myoclonic contractions were observed. Midazolam and phenytoin were administered, but myoclonic contractions continued. Although the midazolam infusion dose was increased to $18 \mathrm{mcg} / \mathrm{kg} / \mathrm{min}$, his seizures could not be controlled and then thiopental infusion was initiated. MRI was reported as normal except hypoxic changes. In the EEG examination under thiopental infusion, very frequent, generalized, lasting $0.5-1 \mathrm{sec}$, high- amplitude spike-slow wave discharges and intermittent bursts were observed. On the $5^{\text {th }}$ day of hospitalization, thiopental infusion was discontinued decreasingly, and then myoclonic seizures reappeared.

On the $10^{\text {th }}$ day of hospitalization, thiopental was reduced again and this once it was observed that myoclonic seizures only increased during the patient's examination and stimulation to the patient and consciousness of the patient improved (GCS:10). The infusions were then discontinued, considering that the patient's current condition was compatible with LAS and piracetam was added to phenobarbital and levetiracetam treatments. On the $60^{\text {th }}$ day of his hospitalization, he was conscious, could sit without support, speak single words. He was transferred from the pediatric intensive care unit.

\section{Discussion}

PHSE is characterized by generalized or multifocal myoclonic contractions and occurs within the first 72 hours of a typical cardiopulmonary arrest and ends within days (Hui et al., 2005). It is thought that it arises from subcortical regions such as brain 
stem. Typically, it is characterized by myoclonic jerks, which can also involve face and neck muscles. Also in our patient, spontaneous myoclonic jerks were generalized and including facial and neck muscles. It continued despite the midazalom infusion until the thiopental infusion was started. Initially, consciousness could not be fully evaluated since his received midazolam and thiopental infusions. PHSE cases are known to be treatment-resistant (Freund et al., 2017). In our patient, myoclonic jerks were partially controlled by thiopental infusion. In the meta-analysis of 65 articles consisting of acute PHSE cases in the literature, 431 cases were reported and EEG findings of these were found to be different. If generalized PHSE cases do not die, vegetative life may develop or they may rarely heal with sequelaes. In some of healing patients may develop LAS (Gupta and Caviness, 2016). Also, in our patient it was detected an improvement in his consciousness within days, and then LAS developed.

LAS was first described in 1963 (Lance and Adams, 1963). Since then, cases have been published, almost all of them in the adult age group. LAS can occur directly after hypoxia and also can be seen in patients who developed PHSE. The form of myoclonus is generalized and multifocal in PHSE, whereas it is action myoclony in LAS. Since our patient was under heavy sedation, we were able to evaluate his state of consciousness after interruption of sedation and infusion of anesthetic drugs. We were only able to diagnose LAS after observing improvement in consciousness and myoclonic jerks, and probably delayed to diagnose it. In treatment, antiepileptic drugs are used because myoclonies are usually of cortex origin. Piracetam, has been found to be useful in the treatment of myoclonies and the efficacy of levetiracetam, which is chemically similar to this agent, has also been reported (Krauss et al., 2001). We found a significant reduction in action myoclonies as a result of the addition of piracetam.

Many hypoxic patients have been kept alive with the developments in pediatric intensive care and the problems which are secondary to hypoxia have been struggled. However, we did not find any cases of PHSE and LAS in childhood. In conclusion, we present this case to emphasize that LAS also can be seen in childhood.

\section{Conflict of interest}

None to declare.

\section{Acknowledgments}

None to declare.

\section{References}

1. Freund, B., Sutter, R., Kaplan, P.W., 2017. Lance-Adams Syndrome in the pretargeted temperature management. Era. Clin. Neurosci. 48(2), 130-138.

2. Gupta, H.V., Caviness, J.N., 2016. Post-hypoxic Myoclonus: Current Concepts, Neurophysiology, and Treatment. Tremor and other hyperkinetic movements (New York, N.Y.), 6, 409.

3. Hui, A.C., Cheng, C., Lam, A., Mok, V., Joynt, G.M., 2005. Prognosis following Post anoxic Myoclonus Status epilepticus. Eur. 54(1), 10-13.

4. Krauss, G.L., Bergin, A., Kramer, R.E., Cho, Y.W., Reich, S.G., 2001. Suppression of post-hypoxic and post-encephalitic myoclonus with levetiracetam. Neurology. 56(3), 411-412.

5. Lance, J.W., Adams, R.D., 1963. The syndrome of intention or action myoclonus as a sequel to hypoxic encephalopathy. Brain. 86, 111-136. 